\title{
Biological Support to Obesity Paradox in Renal Cell Carcinoma: A Review
}

\author{
Ming Li Renge Bu \\ Department of Urology, Shengjing Hospital of China Medical University, Shenyang, China
}

\section{Keywords}

Obesity paradox $\cdot$ Renal cell carcinoma $\cdot$ Target/

immunotherapies · Transcriptomic/genomic signatures

\begin{abstract}
Obesity is a proven risk factor and a debated prognostic factor in renal cell carcinoma (RCC). Termed as an "obesity paradox," the topic has churned controversies, with a few arguing of no true biological association. Suggesting otherwise, a few studies revealed adiposity-induced altered molecular and transcriptomic signatures, at both the systemic and local (tumor and peritumoral adipose tissue) levels, in RCC patients, favoring the paradox. Summarizing such studies suggests of a considerable biological support to adiposity as a promising prognostic factor in RCC patients, although much needs to be clarified before adopting it as a valuable addition to the existing prognostic model. (c) 2020 S. Karger AG, Basel
\end{abstract}

\section{Introduction}

Adiposity, defined as a BMI of $>30 \mathrm{~kg} / \mathrm{m}^{2}$ for the western population and $>27.5 \mathrm{~kg} / \mathrm{m}^{2}$ for Asian population [1], is a hereditary syndrome or a lifestyle-associated worldwide debilitating disease characterized by increased white adipose tissue (WAT) within one's body. Classified under metabolic disorders, epidemiological studies have identified adiposity as the risk factor for several medical conditions, including type 2 diabetes, cardiovascular disorders, dyslipidemia, fatty liver, reproductive dysfunction, and psychiatric disorders [2]. Further, adiposity is identified as the second most common risk factor for cancer development [3]. A review of over 1,000 epidemiological studies have linked adiposity to several cancers, including that of the esophagus, thyroid, breast, liver, gallbladder, pancreas, gastric cardia, colon and rectum, kidney, endometrium, prostate, and ovary [4]. However, several studies suggest a lower mortality risk in obese cancer patients, than in normal weight patients, in some cancer conditions. Termed as "obesity paradox," the topic has drawn interest from several research and clinical groups to resolve this issue in establishing adiposity as a prognostic marker in such cancer conditions [5].

Renal cell carcinoma (RCC) is the most frequently occurring form of kidney cancer, accounting for $90 \%$ of all its diagnosed types. Claiming $2.4 \%$ of all adult cancers, $>400,000$ new RCC cases are diagnosed each year across the world, with $>175,000$ recorded deaths (http://gco. iarc.fr/today/data/factsheets/cancers/29-Kidney-factsheet.pdf). Histologically, RCC is a heterogeneous group of cancers with diverse subtypes, each varying in their clinical course and response to therapy. Clear cell RCC karger@karger.com www.karger.com/uin

(C) 2020 S. Karger AG, Base

Karger"
Renge $\mathrm{Bu}$

Department of Urology, Shengjing Hospital of China Medical University 36 Sanhao Street Heping District

Shenyang, Liaoning 110004 (China)

burg@sj-hospital.org 
(ccRCC) claims about $75 \%$ of these subtypes followed by papillary RCC, which takes up another 15\%; the rest of the $10 \%$ is claimed by chromophobe RCC and other variants. RCC, similar to other cancers, is the result of genetic mutation, the development and progression of which are contributed to by a plethora of biological molecules. The etiological factors contributing to these mutations and molecular mechanisms are only partly understood [6]. Lifestyle risk factors such as tobacco smoking and metabolic factors such as hypertension, type 2 diabetes, and increased triglycerides have been associated with the RCC development $[7,8]$.

Analysis of the results from several studies has established adiposity as a major, but modifiable, risk factor of RCC development [9]. Over the last decade, adiposity has drawn more interest due to its paradoxical influence on the development and prognosis of RCC [10]. Apparently, the topic is controversial, with some groups suggesting several confounding factors, including inadequacy of BMI as a measure of adiposity, in the studies demonstrating survival benefits of obesity in cancer patients [11]. However, a few studies have revealed the molecular and transcriptomic signatures within the tumor and peritumoral adipose tissue between obese and normal weight RCC patients that may possibly favor the survival of obese RCC patients $[12,13]$. These studies suggest a strong need for a better understanding of the underlying molecular mechanisms that may elucidate this obesity paradox in RCC. This prompted us to put together the currently known molecular interactions between the adipose tissue and RCC, which we hope will provide insights into designing and conducting further studies to resolve this paradox.

\section{Adiposity and Risk of Cancer - Molecular Cross-Talk}

WAT is the primary site of storage of excess energy, in the form of triacylglycerol, in our body. This simple storage tissue is converted into a metabolically dynamic organ by its cells harbored within, which include adipocytes; stromal-vascular fraction comprising endothelial cells, pericytes, and adipose precursor cells; and immune cells comprising $\mathrm{T}$ and $\mathrm{B}$ lymphocytes, macrophages, dendritic cells, neutrophils, and mast cells [14]. Through a plethora of biomolecules released by these cells, WAT participates in the regulation of a variety of diverse biological functions at both the local and systemic levels, the latter via blood circulation. In addition to normal biological activities, some of these biomolecules, such as adipokines, pro-inflammatory cytokines, hormones, and fatty acid metabolism regulators, have been postulated to promote obesity-driven cancer risk and progression by altering concerned pathways (Fig. 1) [15, 16].

Leptin and adiponectin are the 2 major adipocyte-released cytokines, the balance between which is proven to be critical in carcinogenesis [17]. In obese individuals, serum leptin levels are increased by 5 - to 10 -fold greater in magnitude than in normal weight individuals [18]. Such increased leptin levels have been associated with the risk of several metabolic diseases, including cancer. Leptin can stimulate the proliferation and promote survival of cancer cells through mitogen-activated protein kinase (MAPK), Jak/Stat, and PI3K/AKT pathways, which are involved in oncogenic signaling, angiogenesis, and immunomodulation. Conversely, a significant reduction in adiponectin levels is observed in obese individuals [19, 20]. Adiponectin, being antagonistic to leptin activity, can exert antitumor effects by inhibiting angiogenesis and decrease macrophage infiltration by stimulating AMPK and caspase activity while suppressing mTOR and Stat3 pathways [21]. Hypoadiponectinemia has been associated with increased tumor formation in the liver in mice, demonstrating the anticancer effect of adiponectin [22]. Thus, decreased levels of adiponectin in obese individuals render them susceptible to cancer development. Ceruloplasmin is another adipokine that is overexpressed in obese individuals [23] and is known to stimulate cancer angiogenesis by SLC31A1-mediated vascular endothelial growth factor (VEGF) production. Adipose fatty acidbinding protein, a recent addition to the adipokine family, has also been linked with carcinogenesis through the interleukin 6 (IL-6)/STAT3/ALDH1 signaling pathway $[24,25]$.

In lean state individuals (BMI 18.5-24.9), the WAT is rich in alternatively activated macrophages, eosinophils, group 2 innate lymphoid cells, invariant natural killer $\mathrm{T}$ cells, T-helper type 2 cells, and regulatory T cells, all of which are associated with the type 2 immune axis [26]. These cells, together with adipose cells, manage the energy balance and exert anti-inflammatory effects via corresponding type 2 (anti-inflammatory) cytokines. However, during obesity, the adipocytes die of hypertrophy, which triggers innate immune response, thus altering the type 2 immune cells to type 1 (pro-inflammatory) state [26]. Now referred to as inflamed-WAT, this is rich in pro-inflammatory cytokines, including TNF- $\alpha$ (the master type 1 cytokine), which is also flushed into systemic circulation via blood influencing the systemic homeostasis. TNF- $\alpha$ can induce the release of other type 1 cytokines and angiogenic factors through cyclooxygenase 2 and prostaglandin E2 production, in turn favoring cancer de- 


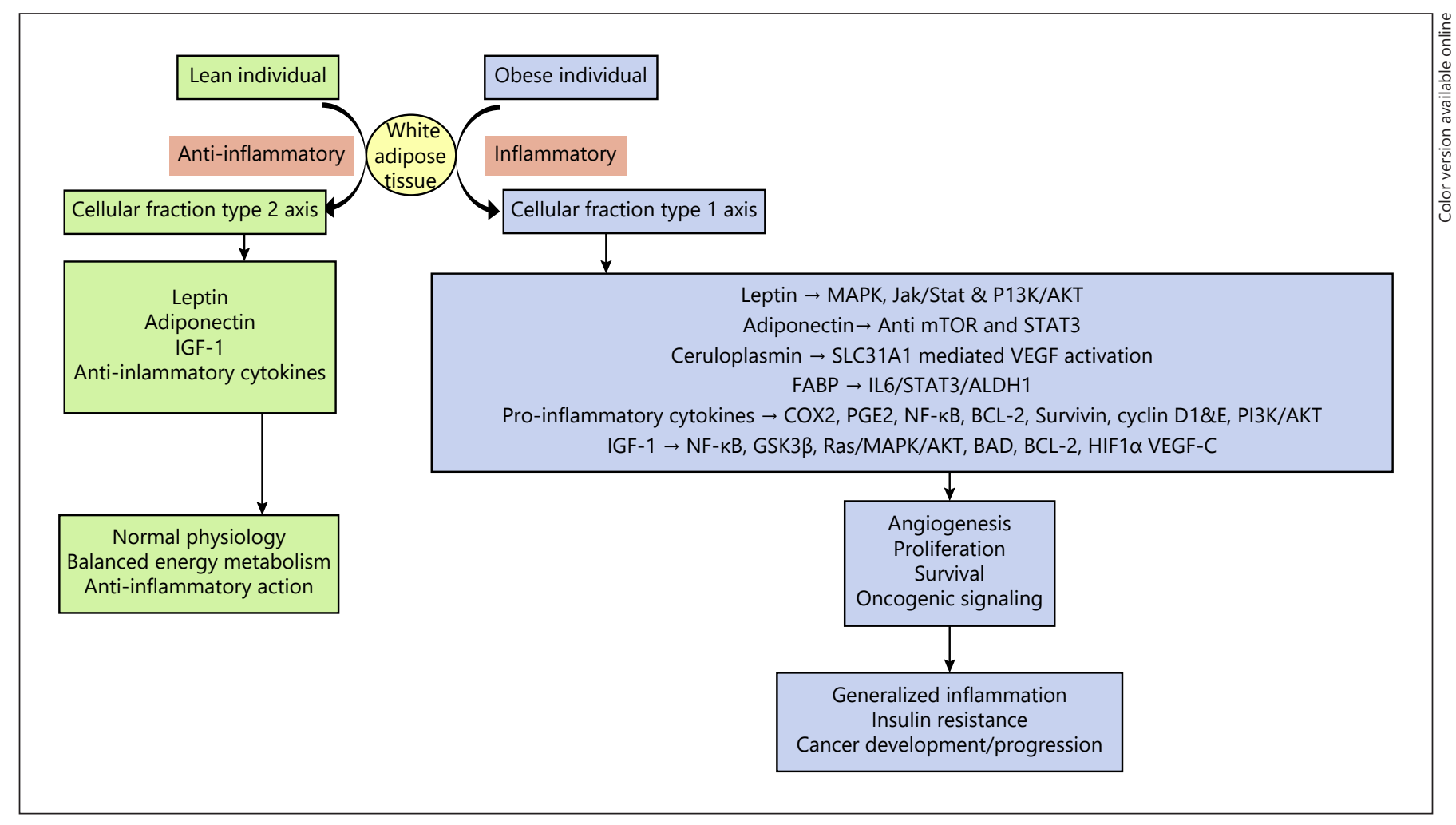

Fig. 1. Flowchart showing the molecular link between adiposity and cancer. In lean individuals, the cellular fraction of the adipose tissue maintains the type 2 axis, an anti-inflammatory lineage, which releases a physiological level of adipokines (leptin and adiponectin) and hormones (IGF-1) along with anti-inflammatory cytokines, which help balance body physiology and energy metabolism. However, in obese individuals, the cellular fraction of the adipose tissue procures the type 1 axis, an inflammatory lineage, which now releases altered levels of adipokines (leptin, adiponectin, ceruloplasmin, and FABP), hormones (IGF-1) along with proinflammatory cytokines, which, by acting through specific pathways, induce angiogenesis and oncogenic signaling, which supports the development, survival, proliferation, and progression of

velopment and progression [27, 28]. Further, through the NF- $\kappa B$ pathway, TNF- $\alpha$ can increase antiapoptotic factors such as BCL-2 and survivin in cancer cells, ensuring their survival and proliferation through the increase of cyclin D1 and cyclin E [15]. Other type 1 cytokines induced by TNF- $\alpha$ can exert antiapoptotic effects on cancer cells, while promoting their proliferation, through the PI3K/AKT pathway.

Obese individuals demonstrate insulin resistance, in response to which there is an early increase in insulin-like growth factor 1 (IGF-1) levels and IGF-1 receptors in WAT and other tissues [29]. IGF-1 is an autocrine/paracrine hormone which is involved in cell survival and pro- cancer cells. IGF-1, insulin-like growth factor-1; FABP, fatty acidbinding protein; MAPK, mitogen-activated protein kinase; Jak/ Stat, Janus kinase/signal transducer and activator of transcription; PI3K/AKT, phosphatidylinositol 3-kinase/protein kinase B; mTOR, mammalian target of rapamycin; SLC31A1, solute carrier family 31 member 1; VEGF, vascular endothelial growth factor; IL-6, interleukin 6; ALDH1, aldehyde dehydrogenase 1; COX2, cyclooxgenase 2; PGE2, prostaglandin E2; NF- $\mathrm{kB}$, nuclear factor kappa-light-chain-enhancer of activated B cells; BCL-2, B-cell lymphoma 2; GSK3 $\beta$, glycogen synthase kinase 3 beta; Ras, retrovirus-associated DNA sequence; BAD, BCL-2-associated agonist of cell death; HIF-1a, hypoxia-inducible factor 1 alpha; WAT, white adipose tissue.

liferation. Through GSK3 $\beta$-mediated NF- $\kappa \mathrm{B}$ activation, IGF-1 exerts prosurvival effects on cancer cells [30]. Further, IGF-1 can block apoptosis of cancer cells through Ras/MAPK/Akt pathway-mediated inactivation of BAD, a proapoptotic molecule, that further prevents Bcl-2 suppression. IGF-1 is also involved in angiogenesis, through HIF-1 $\alpha$ and VEGF-C pathway and by direct action on vascular and lymphatic endothelial cells, which is vital in survival and progression of cancer [31].

Increased incidence of breast cancer in obese women has been noted in several epidemiological studies [32]. The type 1 cytokines and leptin have shown to induce aromatase expression, encoded by the CYP19 gene, in un- 
differentiated adipocytes stimulating estrogen synthesis, which manifests as increased expression in estrogen receptors' target genes. Consequently, this increased biosynthesis of estrogen has been linked to the incidence of estrogen receptor-positive breast cancer in obese women $[32,33]$.

An increased efflux of fatty acids has been observed in obese individuals. The metabolism of this includes uptake, esterification, lipolysis, and mitochondrial oxidation by several regulatory enzymes, including fatty acid synthase (FASN). An alteration in the expression of these regulators has been noted in different cancers and has thus been correlated with their occurrence and progression, the details of which are reviewed in detail elsewhere [34].

\section{Adiposity and RCC - The Paradox}

Epidemiological studies have revealed a correlation between adiposity and RCC in both men and women [35]. A small difference in the relative risk score for RCC and BMI between obese men and women, 1.57 versus 1.72 , respectively, was reported in a meta-analysis of decades of observational studies [36]. Assessing the body size and RCC incidence in a US population, Adams et al. [37] identified a weight gain of $>20 \mathrm{~kg}$ between 18 and 35 years of age and between 35 and 50 years of age, but not after 50 years of age, as a strong correlating factor for RCC occurrence in both men and women. A study by Bertrand et al. [38] also associated obesity with renal mass complexity; however, they specified the need to measure the obesity by waist circumference and not by BMI. From the Metabolic Syndrome and Cancer Project (Me-Can) data, Häggström et al. [8] assessed the association between metabolic factors and the risk of RCC in a large population of 560,388 subjects. The results identified increased $\mathrm{BMI}$ as an independent risk factor for RCC occurrence in women, while increased BMI along with hypertension, and increased glucose and triglyceride levels were separately correlated with RCC occurrence in men. No biological interactions between the assessed metabolic factors were found in the study. Another analysis identified increased fasting insulin levels and, more specifically, diastolic hypertension as the risk factors for RCC in obese individuals [9]. Increased para- and perirenal fat thickness, observed in obese individuals, has been associated with a significant increase in diastolic blood pressure [39], which may partly explain the association between $\mathrm{BMI}$ and diastolic blood pressure as risk factors in RCC occurrence. An exploration into the underlying biology between obesity and RCC reveals several local and systemic mechanisms. Wang et al. [40] assessed the circulat- ing levels of obesity biomarkers and RCC risk, which revealed a significant positive correlation between RCC occurrence and increased circulatory levels of C-peptide, IL-6, and TNF- $\alpha$. A similar trend was also observed in RCC tumor and peri-tumoral microenvironment, along with other molecular changes which are discussed in the respective sections below.

Paradoxically, a study by Kamat and colleagues [41] demonstrated a protective effect of adiposity on RCC patients, resulting in a longer overall survival (OS) of overweight and obese RCC patients, than those with normal weight. Since then, several epidemiological and observational studies and their meta-analysis have confirmed this paradox that obese RCC patients respond better to treatment, measured by the OS and progression-free survival (PFS), than normal weight patients. A multivariate analysis of the data of 1,543 nephrectomized RCC patients revealed a significantly higher OS and cancer-specific survival (CSS) in obese patients than in those with normal BMI [42]. Added in the report was a meta-analysis of the relevant studies, which corroborated that adiposity significantly improved the OS, CSS, and recurrence-free survival, thus claiming preoperative BMI as an independent prognostic indicator for survival among RCC patients. Similarly, BMI was proved to be a prognostic factor for improved OS and PFS in 6,632 metastatic RCC patients who received target therapy as their treatment [43]. However, the topic of obesity paradox is controversial within the concerned community, with a few researchers arguing that such paradox could be the result of certain bias in the research design of the studies, such as reverse causation bias and selection bias, rather than a true biological association [44].

A recent study by Sanchez et al. [13] analyzed 478 ccRCC patients from 3 independent clinical cohorts: (1) COMPARZ phase 3 clinical trial cohort - anti-VEGF therapy-treated metastatic ccRCC patients, (2) Memorial Sloan Kettering (MSK) immunotherapy cohort - immunotherapy-treated metastatic ccRCC patients, and (3) The Cancer Genome Atlas (TCGA) surgery cohort - nephrectomy-treated nonmetastatic ccRCC patients. The results revealed a significantly longer OS of obese patients, than those with normal BMI, in both COMPARZ and TCGA cohorts, but not in the MSK cohort [13]. This study further associated the transcriptomic profiles with the patient survival data and found a few vital information that may favor the obesity paradox. Previously, a few other studies have also attempted to elicit the genetic/molecular mechanisms underlying this obesity paradox, the results of which are discussed below. 
Tumor and Peritumoral Fat Microenvironment in

\section{Normal BMI versus Obese RCC Patients}

Considering the controversy around the obesity paradox, efforts are being made to elicit any possible biological association between adiposity and RCC prognosis. This is mostly carried out by looking for genetic/molecular changes within the RCC tumor and the peritumoral environment, mainly PAT. PAT is said to contain dormant brown adipose tissue (BAT) [45], and increased expression of UCP-1, a protein specific to BAT, in PAT indicates poor prognosis in RCC patients [46]. Zi et al. [47] identified an overexpression of multiple WNTs in both stage-3 RCC tumor and in part of the PAT which is in close proximity to the tumor, referred to as perineoplasm adipose tissue. Further, the authors demonstrated that the conditioned medium from perineoplasm adipose tissue increased the migration of kidney cancer cells, in vitro, through increased WNT/ $\beta$-catenin activity. Considered as a metabolic oncogene, FASN has been associated with poor prognosis in several cancers, including RCC [12]. In lieu with this, the analysis of "The Cancer Genome Atlas" data set and immunohistochemistry revealed a significant decrease in the FASN gene expression in obese RCC patients, compared to that in those with normal BMI $(p=0.034)$. Consistently, the low FASN-expressing RCC patients in this study demonstrated an OS of 36.8 months (median), which was significantly longer than that in their normal BMI counterparts who survived only for about 15 months (median) $(p=0.002)$ [43].

As previously stated, the serum concentration of adiponectin, an adipocyte-released adipokine, is reduced in obese individuals, who are thus prone to increased risk of cancer occurrence, including RCC. However, assessment of adiponectin levels in PAT by Ito et al. [48] showed no significant difference between healthy individuals and RCC patients. However, among the RCC patients, the adiponectin levels within PAT were lower in obese patients than in normal BMI patients. Further, the team also noted a significant decrease in the expression of adiponectin receptors, AdipoR1 and AdipoR2, within the RCC tumor tissue, compared to that in the surrounding normal parenchyma, with no variation between obese and normal BMI patients. However, this variation in adiponectin levels within PAT and decreased AdipoR1/R2 within tumors failed to correlate with the RCC aggressiveness and OS for both obese and normal BMI RCC patients. Studies have associated hypermethylation of several genes to renal carcinogenesis and prognosis [49, 50]. Identified among such genes are the obesity-related genes, neuropeptide $\mathrm{Y}$, leptin, and leptin receptor, which are significantly hyper-

Biological Support to Obesity Paradox in RCC methylated compared to the normal adjacent parenchyma tissue $(p<0.0001)$ [51]. Further, hypermethylation of the leptin receptor gene, which may reduce the corresponding protein expression, was associated with increased risk of disease recurrence. The authors speculate the underlying mechanism to involve the ability of the leptin receptor gene to exert antimetastatic effects via Stat3-mediated activation of matrix metalloproteinase enzymes, which might be lost due to hypermethylationassociated reduction in leptin receptor expression. The genotyping of Gln223Arg polymorphism rs1137101 at the leptin receptor gene has also been studied in RCC patients [52]. The results provide a strong association of GG genotype with more aggressive tumor and shorter OS in RCC patients, compared to GA and AA genotypes. Considering up to a 10 -fold increase in systemic circulation of leptin in obese individuals, a local alteration in leptin and leptin receptor genes within the RCC tumor may play a crucial role in RCC tumor physiology, which needs further investigation. Increased expression of other obesityrelated genes, those involved in insulin signaling, IL-6 signaling, and TNF signaling pathways, has also been identified in the TCGA database and associated with obesity-driven RCC [40]. The interaction of these genetic alterations with the obesity-induced systemic inflammation and insulin resistance needs to be clarified in future studies. Shen et al. [53] identified an increased number of type 2 (M2) macrophages within the PAT of metastatic RCC patients. A previous study has shown that when cocultured, RCC cells can induce M2 phenotype in macrophages [54]. So, whether these increased M2 macrophages seen in the PAT of RCC patients were the result of a cross talk between RCC tumor and innate macrophage pool within the PAT, or whether these were M2 macrophages recruited from an external source needs further investigation. Further, the effect of obesity on this M2 macrophage population also needs to be assessed. Either way, RCC may be at advantage of being in proximity of M2 macrophages within PAT, as these immune cells are known to promote tumor progression through angiogenesis via releasing angiogenic factors, cytokines, and matrix metalloproteinase enzymes [55].

Recently, Sanchez and colleagues [13] exclusively studied transcriptomic signatures related to the obesity paradox in both the tumor tissue and PAT of ccRCC patients who underwent immunotherapy or surgical intervention. In general, compared to normal BMI patients, tumors from obese patients overexpressed wound healing (TGF- $\beta$, epithelial-mesenchymal transition, hedgehog, and notch), hypoxia, and angiogenesis (VEGF and 
Fig. 2. Variation in cellular fraction within the tumor and peritumoral microenvironment in obese versus nonobese metastatic RCC patients. RCC, renal cell carcinoma.

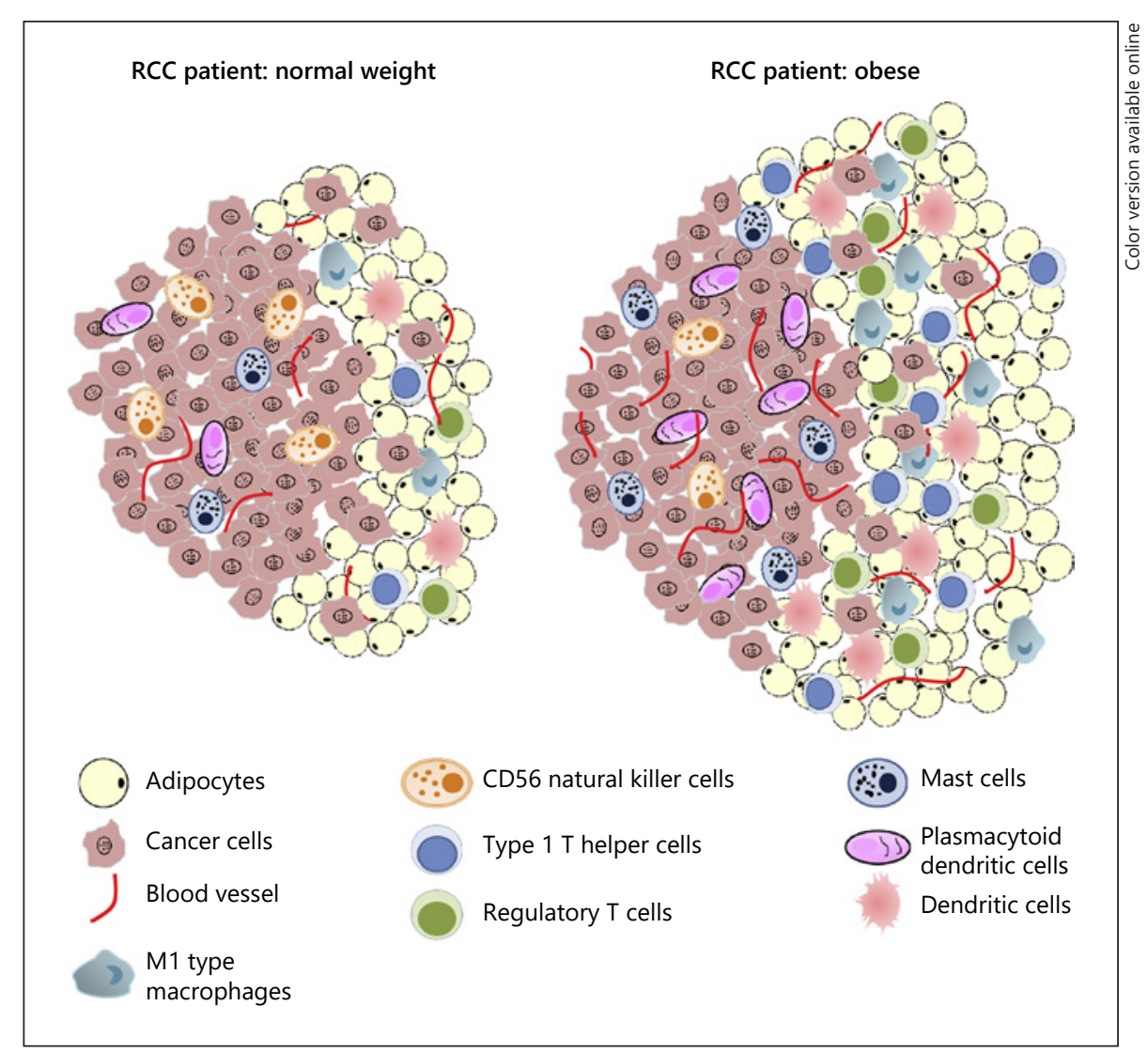

VEGF receptor 2) signaling pathways, along with the metabolic pathways, involved in adipogenesis, glycolysis, and fatty acid metabolism. Surprisingly, the ccRCC tumor from obese patients revealed a downregulation in interferon (IFN) $-\gamma$ pathway, with no changes in other inflammatory pathways, letting the authors conclude that the ccRCC tumor in obese patients is not high on local inflammation. A low expression of several immune checkpoint molecules, including PD-L1, was also noted in obese patient tumor samples compared to those from normal BMI patients. The tumors from obese patients had a similar immune cell fraction as normal weight individuals, except for an increase in plasmacytoid dendritic cells and mast cells, and decreased CD56+ natural killer cells (Fig. 2). In addition to increased hypoxia and angiogenesis pathways, the PAT of obese ccRCC patients demonstrated a significant increase in several canonical inflammatory signatures, including TNF- $\alpha$, IFN- $\alpha$, IFN- $\gamma$, Th1, and Th2 pathways; CD28 signaling in Thelper cells; and dendritic cell maturation, compared to normal BMI patients. A pronounced increase in the immune cell infiltration, including type $1 \mathrm{~T}$-helper cells, regulatory $\mathrm{T}$ cells, dendritic cells, and type 1 macrophages, was also shown in the PAT of obese ccRCC patients, compared to their normal weight counterparts (Fig. 2).

\section{Response to RCC Treatment: Obese versus Normal Weight Patients}

While surgery remains the preferred mode of treatment for localized or locally advanced ccRCC, those with metastasis are treated with targeted therapy or immune checkpoint inhibitor therapy. The results of several studies indicate a prognostic role of obesity in both these treatment groups, which are discussed below (Fig. 3).

\section{Nephrectomy-Treated RCC Patients}

Two early studies contradicted their opinion on the prognostic value of BMI in surgically treated RCC patients. A multivariate regression analysis of data from 400 surgically treated RCC patients claimed BMI to serve as a good predictor of disease-specific death, with overweight and obese patients demonstrating favorable prognosis [41]. However, a consecutive study by Donat and colleagues [56] failed to establish the impact of BMI on OS and PFS of nephrectomized RCC patients. Since then, several studies have been conducted that show a positive correlation 
Fig. 3. A summary of obesity paradox in RCC patients: obesity is associated with increased risk of RCC and is associated with decreased OS if left untreated. However, when treated surgically or with targeted therapies, obese RCC patients demonstrates significantly longer OS compared to normal-weight patients. RCC, renal cell carcinoma; OS, overall survival; IFN- $\gamma$, interferon- $\gamma$.

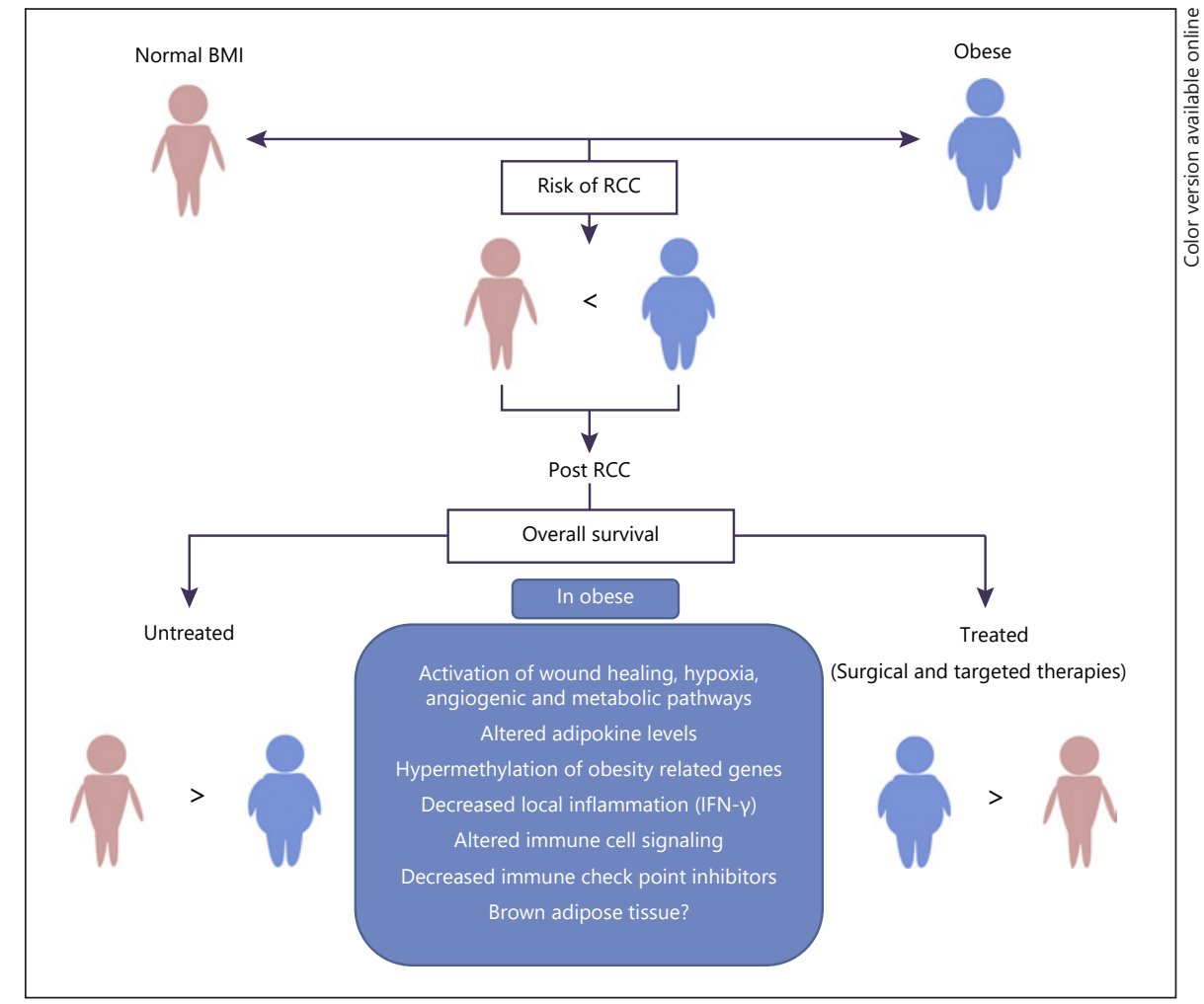

between adiposity and post-nephrectomy prognosis in RCC patients. A study in 1,017 Korean patients with RCC, who underwent curative surgery as their treatment, showed an improved OS of overweight and obese RCC patients compared to normal weight patients, $p<0.04$ and $<0.022$, respectively [57]. In partial nephrectomy-treated 1,338 ccRCC patients, Waalkes et al. [58] observed a gradual increase in the 5-year tumor-specific survival rate with respect to patients' BMI; $70.9 \%$ for pre-obese, $74.0 \%$ in grade I obese, and $85.6 \%$ in grade II obese as opposed to $63.8 \%$ in low BMI patients $(p<0.001)$. Owing to the sufficient data available by then, a meta-analysis was carried out by Choi et al. [42], which associated presurgery high BMI in RCC patients with improved OS, CSS, and recurrence-free survival when compared to low BMI patients, and concluded BMI as an independent prognostic indicator for survival in surgically treated RCC patients [39]. Although Rogde and group [59] confirmed the accelerating protective effects of BMI in surgically treated RCC patients, they reported a significant increase in the postoperative complications translating into increased need of intermediate/ ICU care for obese patients. A recent meta-analysis revealed an increase in mortality, and a decrease in hazard ratio, by $5 \%$ for each $1 \mathrm{~kg} / \mathrm{m}^{2}$ increment of BMI in RCC patients [60]. As a conclusion, the study associated adipos-

Biological Support to Obesity Paradox in RCC ity with increased mortality in whole RCC patients and a better survival in surgically treated RCC patients, depicted in Figure 3. Analysis of data from the TCGA cohort by Sanchez et al. [13] also confirmed a direct correlation between BMI and OS of surgically treated RCC patients after adjusting for stage or grade of the disease.

\section{Targeted/Immunotherapy-Treated RCC Patients}

Currently approved targeted therapy for metastatic RCC involves drugs that primarily target angiogenesis. The drugs can be classified into monoclonal antibodies (bevacizumab - binds and neutralizes VEGF-A) and tyrosine kinase inhibitors (TKIs), which can be further divided into VEGF receptor inhibitors (sunitinib, pazopanib, sorafenib, cabozantinib, axitinib, lenvatinib and tivozanib) and mTOR inhibitors (everolimus and temsirolimus). Immunotherapies involve immunocheckpoint inhibitors targeting programmed death-1 (PD-1) (nivolumab and pembrolizumab) and its ligand (PD-L1) (atezolizumab and avelumab) and cytotoxic T-lymphocyte antigen 4 (ipilimumab).

In 2010, Choueiri et al. [61] published a first large report demonstrating a protective effect of adiposity on ccRCC patients, treated with first-line anti-VEGF therapy. The majority of the patients in this study were treated 
with sunitinib (61\%), followed by sorafenib (30\%) and bevacizumab (9\%), the combined data of which showed a longer OS of obese (BMI $\left.>30 \mathrm{~kg} / \mathrm{m}^{2}\right)$ patients over normal BMI patients. Steffens et al. [62] identified a positive correlation between high visceral fat area (VFA) and subcutaneous fat area with longer OS and PFS in metastatic RCC patients who received antiangiogenic targeted therapy (sunitinib, sorafenib, axitinib, and bevacizumab). In contrast, VFA and subcutaneous fat area were associated with shorter OS and total time to progression when the assessed group of metastatic RCC patients involved those who received antiangiogenic targeted therapy and cytokine therapy [63]. Another study involving a similar treatment group of metastatic RCC patients, but with fewer number of patients treated with cytokines, was not only able to establish a significant association of between VFA and improved OS and PFS, but also claimed VFA to improve the prognostic value of Memorial Sloan Kettering Cancer Center (MSKCC) classification in predicting the OS in such patients [64]. Choueiri et al. [61] assessed the impact of BMI and OS in 2 large cohorts of metastatic RCC patients treated with TKIs, the results of which favored the positive correlation between BMI and OS in both groups, even when matched with the International Metastatic Renal Cell Carcinoma Database Consortium (IMDC) criteria [43]. Later, the same group was able to associate both BMI and body surface area as markers of obesity to predict an improved outcome in metastatic RCC patients treated with VEGF-targeted therapies [61]. A recent study was able to demonstrate BMI as a prognostic marker for longer OS in metastatic RCC patients treated specifically with cabozantinib [65]. The data from metastatic ccRCC patients from the COMPARZ clinical trial, who received sunitinib or pazopanib as their treatment, were assessed by 2 studies for identification of BMI as at least one of the predictors of the outcome of TKI therapy. The study by Hakimi confirmed a strong correlation between BMI and OS of the assessed cohort but noted a stronger correlation in the sunitinib-treated group than the pazopanib group [66]. This trend was also reported in the subsequent study by Sanchez and team [13], confirming a stronger association of BMI with sunitinib in metastatic RCC patients. Previously, a combination of atezolizumab and bevacizumab was shown to result in better clinical benefit than sunitinib in RCC patients [67]. However, it is not clear if BMI can predict the patient subgroups who would benefit from a particular targeted therapy [10].

Recent studies on BMI as a prognostic marker in immunotherapy-treated metastatic RCC patients have dis- closed mixed results. A retrospective analysis of 976 advanced RCC patients, treated with PD-1/PD-L1 inhibitors, demonstrated a significant increase in OS, PFS, and time to treatment failure in overweight and obese patients compared to normal weight patients [68]. Although these results were uniform across both sex, a gender bias was noted for PFS in male patients $(p=0.0668)$, indicating a possible role of altered molecular mechanisms between genders. A similar trend was reported by Lalani et al. [69] where the BMI in immunotherapytreated, with or without other targeted therapy, metastatic RCC patients was significantly correlated with a longer OS, while the PFS failed to reach significance, but this time in both sexes. Further, the study also revealed that BMI reduction, from normal to low, during immunotherapy correlated with much shorter OS than in those with no BMI changes. Assessment of a large population of nivolumab-treated metastatic RCC patients by De Giorgi et al. [70] established a correlation between low BMI $\left(<25 \mathrm{~kg} / \mathrm{m}^{2}\right)$ and shorter OS. Further, when combined with an increased systemic inflammation score $(>1.375)$, low BMI was associated with worst OS in these patients. In the MSK immunotherapy cohort, the median OS of obese patients was 49.9 months as compared to 15.6 months in normal weight patients [13]. However, the difference failed to reach statistical significance after adjusting for IMDC risk score. As a contradiction, an inverse trend was reported by Bergerot et al. [71], where a small group of immunotherapy-treated metastatic RCC patients with low BMI had a median OS of 23.6 months compared to 19.9 months in overweight/obese patients. A study by Labadie et al. [72] reported that BMI was not correlated with OS in ccRCC patients with primary resistance to immunotherapy, while the correlation was positive in immunotherapy-sensitive patients.

The Sanchez study [13] further revealed a few interesting molecular changes within the tumor microenvironment between obese and normal weight patients in the COMPARZ (targeted therapy-treated RCC patients) cohort. Primarily, obese patients demonstrated a higher proportion of infiltrating plasmacytoid dendritic cells within their tumor microenvironment, than normal weight patients. Further, a lower expression of several immune checkpoint molecules and PD-L1 was evident in obese patients than in normal weight individuals. Of note, such changes were not observed in the TCGA cohort (surgically treated RCC patients), suggesting of the role of targeted therapy in eliciting such molecular changes. 


\section{Discussion}

All the clinical data concerning adiposity and RCC discussed above generally indicate survival benefit of obese over normal weight patients, despite the type of treatment received. Initial controversies have led to define the relationship between adiposity and RCC as an "obesity paradox," with many groups suggesting of several confounding factors impacting the results of such analysis, with no actual biological correlation. Although the World Health Organization uses BMI to define overweight and obesity, using BMI as a surrogate marker of adiposity is flawed due to its inability to identify sarcopenic obesity, a condition with high body fat mass with low muscle mass. In this regard, studies assessing sarcopenic obesity instead of BMI as the prognostic factor demonstrated a significant decrease in OS and PFS in sarcopenic RCC patients compared to normal weight patients [73-75]. These studies suggest the need to use radiologically measured subcutaneous, visceral, intra-, and intermuscular adipose tissue as the measures of adiposity instead of BMI for more accurate results. Further, although such procedures might be cumbersome to follow, it would be interesting to see how their results may affect the obesity paradox [10]. Even if BMI is proved prognostic, its contribution to the current prognostic model is unclear. However, considering results of some studies claiming that change in BMI during the treatment can change OS of the RCC patients, 64 addition of adiposity as a prognostic measure may carry clinical value. However, still there are several unanswered questions, some of which are discussed below, that need to be clarified before its clinical translation as a prognostic factor.

Adiposity-induced altered adipokine levels, primarily of leptin and adipokine, have been associated with carcinogenesis of several types of cancer, but not with RCC [17, 76]. Hypermethylation of leptin/leptin receptor gene and adipokine receptor gene within the RCC tumor may have a role to play in this but needs further studies. Usually, obesity and cancer are associated with enriched inflammatory signatures. Although PAT from obese ccRCC patients was enriched with proinflammatory molecules, their RCC tumor was not high on local inflammation. The role of this discrepancy in the obesity paradox needs further investigation. As noted above, increased expression of the BAT-associated protein UCP-1 in PAT is negatively associated with RCC prognosis [46]. Previously, studies have associated decreased BAT and UCP-1 activity with weight gain in breast cancer patients and high-fat-fed murine models, respectively $[77,78]$. Thus, it will be interesting to further elicit the role of brown fat in the RCC-associated obesity paradox. FASN, a risk factor in RCC, is inversely correlated with BMI in RCC patients. Understanding the mechanism of how adiposity regulates FASN in RCC patients may elicit interesting pathways that may assist in future target therapies. Any possible role of higher proportion of plasmacytoid dendritic cells and mast cells within the RCC tumor, and enriched immune cell infiltration within the PAT, of obese metastatic RCC patients and their prolonged OS need to be revealed. It is of interest to see if this immune reservoir within the PAT can be further exploited in targeted therapies, like mobilizing these cells into the tumor to elicit an antitumor response. Further, how activated wound healing/hypoxia/angiogenic pathways within the tumor of obese RCC patients contribute to their prolonged OS needs to be revealed. Although sunitinib and pazopanib are both anti-VEGF TKIs, compared to pazopanib, sunitinib demonstrated a stronger correlation between BMI and OS in RCC patients; understanding the mechanisms of this will be of assistance in developing better targeted therapies. Further, the fact that patients' sensitiveness to immunotherapy may alter the OS in obese patients demands the need for careful selection of patients for such therapies. More studies are also needed to assess the effect of change in adiposity during target/immunotherapies on the OS of RCC patients. The study by Rogde et al. [59] revealed the need for increased intermediate/ICU care in surgically treated obese RCC patients, despite demonstrating longer CSS. This raises the question on the quality of life in these patients, wondering if this longer survival is worth the pain, demanding further studies in this regard.

\section{Conclusion}

In summary, in addition to being a risk factor for RCC, adiposity seems to be a promising prognostic factor in obese patients. Results from molecular/transcriptomic analysis have provided biological support to this paradox and have improved our understanding of the different subgroups of RCC patients and the underlying molecular play. However, much needs to be achieved before adopting adiposity to predict these patient subgroups and serve as a valuable addition to the existing prognostic models.

\section{Acknowledgements}

This work was supported by the Promising Talents Plan Program Founding of Shengjing Hospital, China Medical University. We apologize to those whose study we could not cite due to the limitations of our topic and space. 


\section{Conflict of Interest Statement}

There are no conflicts of interest.

\section{Author Contributions}

Ming Li: project development, data collection, manuscript writing, and editing. Renge Bu: project development, data collection, manuscript writing, and editing.

\section{References}

1 WHO Expert Consultation. Appropriate body-mass index for Asian populations and its implications for policy and intervention strategies. Lancet. 2004 Jan 10;363(9403): $157-63$.

2 Kyrou I, Randeva HS, Tsigos C, Kaltsas G, Weickert MO. Clinical problems caused by obesity. In: Feingold KR, Anawalt B, Boyce A, Chrousos G, Dungan K, Grossman A, et al., editors. Endotext. South Dartmouth (MA): MDText.com, Inc.; 2000.

3 Budny A, Grochowski C, Kozłowski P, Kolak A, Kamińska M, Budny B, et al. Obesity as a tumour development triggering factor. Ann Agric Environ Med. 2019 Mar 22;26(1):1323.

4 Lauby-Secretan B, Scoccianti C, Loomis D, Grosse Y, Bianchini F, Straif K. Body fatness and cancer: viewpoint of the IARC working group. N Engl J Med. 2016 Aug 25;375(8): 794-8.

5 Lee DH, Giovannucci EL. The obesity paradox in cancer: epidemiologic insights and perspectives. Curr Nutr Rep. 2019 Sep;8(3): $175-81$.

6 Siegel RL, Fedewa SA, Miller KD, GodingSauer A, Pinheiro PS, Martinez-Tyson D, et al. Cancer statistics for Hispanics/Latinos, 2015, 2019. CA Cancer J Clin. 2019 Jan;65(6): 457-80.

7 McGuire BB, Fitzpatrick JM. BMI and the risk of renal cell carcinoma. Curr Opin Urol. 2011 Sep;21(5):356-61.

8 Häggström C, Rapp K, Stocks T, Manjer J, Bjørge T, Ulmer H, et al. Metabolic factors associated with risk of renal cell carcinoma. PLoS One. 2013;8(2):e57475.

9 Johansson M, Carreras-Torres R, Scelo G, Purdue MP, Mariosa D, Muller DC, et al. The influence of obesity-related factors in the etiology of renal cell carcinoma-A mendelian randomization study. PLoS Med. 2019 Jan; 16(1):e1002724.

10 Gan CL, Heng DYC. New insights into the obesity paradox in renal cell carcinoma. Nat Rev Nephrol. 2020 May;16(5):253-4.

11 Baracos VE, Arribas L. Sarcopenic obesity: hidden muscle wasting and its impact for survival and complications of cancer therapy. Ann Oncol. 2018 Feb 1;29(Suppl_2):ii19.

12 Horiguchi A, Asano T, Asano T, Ito K, Sumitomo M, Hayakawa M. Fatty acid synthase over expression is an indicator of tumor aggressiveness and poor prognosis in renal cell carcinoma. J Urol. 2008 Sep; 180(3):1137-40
13 Sanchez A, Furberg H, Kuo F, Vuong L, Ged Y, Patil S, et al. Transcriptomic signatures related to the obesity paradox in patients with clear cell renal cell carcinoma: a cohort study. Lancet Oncol. 2020 Feb;21(2):283-93.

14 Coelho M, Oliveira T, Fernandes R. Biochemistry of adipose tissue: an endocrine organ. Arch Med Sci. 2013 Apr 20;9(2):191-200.

15 Aurilio G, Piva F, Santoni M, Cimadamore A, Sorgentoni G, Lopez-Beltran A, et al. The role of obesity in renal cell carcinoma patients: clinical-pathological implications. Int J Mol Sci. 2019 Nov 13;20(22):5683.

16 Quail DF, Dannenberg AJ. The obese adipose tissue microenvironment in cancer development and progression. Nat Rev Endocrinol. 2019 Mar; 15(3):139-54.

17 Grossmann ME, Cleary MP. The balance between leptin and adiponectin in the control of carcinogenesis: focus on mammary tumorigenesis. Biochimie. 2012 Oct;94(10):2164-71.

18 Considine RV, Sinha MK, Heiman ML, Kriauciunas A, Stephens TW, Nyce MR, et al. Serum immunoreactive-leptin concentrations in normal-weight and obese humans. N Engl J Med. 1996 Feb 1;334(5):292-5.

19 Arita Y, Kihara S, Ouchi N, Takahashi M, Maeda K, Miyagawa J, et al. Paradoxical decrease of an adipose-specific protein, adiponectin, in obesity. Biochem Biophys Res Commun. 1999 Apr 2;257(1):79-83

20 Horiguchi A, Ito K, Sumitomo M, Kimura F, Asano T, Hayakawa M. Decreased serum adiponectin levels in patients with metastatic renal cell carcinoma. Jpn J Clin Oncol. 2008 Feb; 38(2):106-11.

21 Park J, Morley TS, Kim M, Clegg DJ, Scherer PE. Obesity and cancer: mechanisms underlying tumour progression and recurrence. Nat Rev Endocrinol. 2014 Aug;10(8):455-65.

22 Kamada Y, Matsumoto H, Tamura S, Fukushima J, Kiso S, Fukui K, et al. Hypoadiponectinemia accelerates hepatic tumor formation in a nonalcoholic steatohepatitis mouse model. J Hepatol. 2007 Oct;47(4):556-64.

23 Arner E, Forrest AR, Ehrlund A, Mejhert N, Itoh $\mathrm{M}$, Kawaji $\mathrm{H}$, et al. Ceruloplasmin is a novel adipokine which is overexpressed in adipose tissue of obese subjects and in obesityassociated cancer cells. PLoS One. 2014;9(3): e80274.

24 Greenhill C. A-FABP links obesity and breast cancer. Nat Rev Endocrinol. 2018 Oct;14(10): 566.
25 Hao J, Zhang Y, Yan X, Yan F, Sun Y, Zeng J, et al. Circulating adipose fatty acid binding protein is a new link underlying obesity-associated breast/mammary tumor development. Cell Metab. 2018 Nov 6;28(5):689-705.

26 Brestoff JR, Artis D. Immune regulation of metabolic homeostasis in health and disease. Cell. 2015 Mar 26;161(1):146-60.

27 Maihöfner C, Charalambous MP, Bhambra $\mathrm{U}$, Lightfoot T, Geisslinger G, Gooderham NJ. Expression of cyclooxygenase-2 parallels expression of interleukin-1beta, interleukin-6 and NF-kappaB in human colorectal cancer. Carcinogenesis. 2003 Apr;24(4):66571.

28 Osório-Costa F, Carvalheira JBC. TNF- $\alpha$ in obesity-associated colon cancer. Transl Gastrointest Cancer. 2013;2(4):179-93.

29 Westley RL, May FE. A twenty-first century cancer epidemic caused by obesity: the involvement of insulin, diabetes, and insulinlike growth factors. Int J Endocrinol. 2013; 2013:632461.

30 Ougolkov AV, Bone ND, Fernandez-Zapico ME, Kay NE, Billadeau DD. Inhibition of glycogen synthase kinase- 3 activity leads to epigenetic silencing of nuclear factor kappaB target genes and induction of apoptosis in chronic lymphocytic leukemia B cells. Blood. 2007;110(2):735-42.

31 Björndahl M, Cao R, Nissen LJ, Clasper S, Johnson LA, Xue Y, et al. Insulin-like growth factors 1 and 2 induce lymphangiogenesis in vivo. Proc Natl Acad Sci U S A. 2005;102(43): 15593-8.

32 Chen SI, Hsieh CC. Why are women with obesity more likely to develop breast cancer. Future Oncol. 2018 Jul;14(16):1523-6.

33 Howe LR, Subbaramaiah K, Hudis CA, Dannenberg AJ. Molecular pathways: adipose inflammation as a mediator of obesity-associated cancer. Clin Cancer Res. 2013 Nov 15 19(22):6074-83.

34 Balaban S, Lee LS, Schreuder M, Hoy AJ. Obesity and cancer progression: is there a role of fatty acid metabolism? Biomed Res Int. 2015; 2015:274585

35 Setiawan VW, Stram DO, Nomura AM, Kolonel LN, Henderson BE. Risk factors for renal cell cancer: the multiethnic cohort. Am J Epidemiol. 2007 Oct 15;166(8):932-40.

36 Dobbins M, Decorby K, Choi BC. The association between obesity and cancer risk: a meta-analysis of observational studies from 1985 to 2011. ISRN Prev Med. 2013;2013: 680536 . 
37 Adams KF, Leitzmann MF, Albanes D, Kipnis V, Moore SC, Schatzkin A, et al. Body size and renal cell cancer incidence in a large US cohort study. Am J Epidemiol. 2008 Aug 1; 168(3):268-77.

38 Bertrand LA, Thomas LJ, Li P, Buchta CM, Boi SK, Orlandella RM, et al. Obesity as defined by waist circumference but not body mass index is associated with higher renal mass complexity. Urol Oncol. 2017 Nov; 35(11):661.e1-e6.

39 De Pergola G, Campobasso N, Nardecchia A, Triggiani V, Caccavo D, Gesualdo L, et al. Para- and perirenal ultrasonographic fat thickness is associated with 24-hours mean diastolic blood pressure levels in overweight and obese subjects. BMC Cardiovasc Disord. 2015 Sep 30;15:108.

40 Wang Q, Tu H, Zhu M, Liang D, Ye Y, Chang DW, et al. Circulating obesity-driven biomarkers are associated with risk of clear cell renal cell carcinoma: a two-stage, case-control study. Carcinogenesis. 2019 Oct 16; 40(10):1191-7.

41 Kamat AM, Shock RP, Naya Y, Rosser CJ, Slaton JW, Pisters LL. Prognostic value of body mass index in patients undergoing nephrectomy for localized renal tumors. Urology. 2004 Jan;63(1):46-50.

42 Choi Y, Park B, Jeong BC, Seo SI, Jeon SS, Choi HY, et al. Body mass index and survival in patients with renal cell carcinoma: a clinical-based cohort and meta-analysis. Int J Cancer. 2013 Feb 1;132(3):625-34.

43 Albiges L, Hakimi AA, Xie W, McKay RR, Simantov R, Lin X, et al. Body mass index and metastatic renal cell carcinoma: clinical and biological correlations. J Clin Oncol. 2016 Oct 20;34(30):3655-63.

44 Wilson KM, Cho E. Obesity and kidney cancer. Recent Results Cancer Res. 2016;208:8193.

45 Jespersen NZ, Feizi A, Andersen ES, Heywood S, Hattel HB, Daugaard S, et al. Heterogeneity in the perirenal region of humans suggests presence of dormant brown adipose tissue that contains brown fat precursor cells. Mol Metab. 2019 Jun;24:30-43.

46 Li X, Wang G, Liu J, Ding G. Increased UCP1 expression in the perirenal adipose tissue of patients with renal cell carcinoma. Oncol Rep. 2019 Nov;42(5):1972-80.

47 Zi X, Lusch A, Blair CA, Okhunov Z, Yokoyama NN, Liu S, et al. Effect of perineoplasm perinephric adipose tissues on migration of clear cell renal cell carcinoma cells: a potential role of WNT signaling. Oncotarget. 2016 Aug 16;7(33):53277-88.

48 Ito R, Narita S, Huang M, Nara T, Numakura $\mathrm{K}$, Takayama K, et al. The impact of obesity and adiponectin signaling in patients with renal cell carcinoma: a potential mechanism for the "obesity paradox". PLoS One. 2017;12(2): e0171615.
49 Hu CY, Mohtat D, Yu Y, Ko YA, Shenoy N, Bhattacharya S, et al. Kidney cancer is characterized by aberrant methylation of tissue-specific enhancers that are prognostic for overall survival. Clin Cancer Res. 2014 Aug 15; 20(16):4349-60.

50 Peters I, Gebauer K, Dubrowinskaja N, Atschekzei F, Kramer MW, Hennenlotter J, et al. GATA5 CpG island hypermethylation is an independent predictor for poor clinical outcome in renal cell carcinoma. Oncol Rep. 2014 Apr;31(4):1523-30.

51 Mendoza-Pérez J, Gu J, Herrera LA, Tannir NM, Zhang S, Matin S, et al. Prognostic significance of promoter $\mathrm{CpG}$ island methylation of obesity-related genes in patients with nonmetastatic renal cell carcinoma. Cancer. 2017 Sep 15;123(18):3617-27.

52 Abdu Allah AM, El-Hefnway SM, Alhanafy AM, Zahran AM, Kasem HE. Leptin receptor gene $(A / G)$ polymorphism rs1137101 and renal cell carcinoma. Mol Cell Biochem. 2018 Nov;448(1-2):137-44.

53 Shen K, Vesey DA, Hasnain SZ, Zhao KN, Wang $\mathrm{H}$, Johnson DW, et al. A cost-effective three-dimensional culture platform functionally mimics the adipose tissue microenvironment surrounding the kidney. Biochem Biophys Res Commun. 2020 Feb 12;522(3):73642.

54 Komohara Y, Hasita H, Ohnishi K, Fujiwara Y, Suzu S, Eto M, et al. Macrophage infiltration and its prognostic relevance in clear cell renal cell carcinoma. Cancer Sci. 2011 Jul; 102(7):1424-31.

55 Kovaleva OV, Samoilova DV, Shitova MS, Gratchev A. Tumor associated macrophages in kidney cancer. Anal Cell Pathol. 2016;2016: 9307549.

56 Donat SM, Salzhauer EW, Mitra N, Yanke BV, Snyder ME, Russo P. Impact of body mass index on survival of patients with surgically treated renal cell carcinoma. J Urol. 2006 Jan; 175(1):46-52.

57 Jeon HG, Jeong IG, Lee JH, Lee CJ, Kwak C, $\mathrm{Kim} \mathrm{HH}$, et al. Prognostic value of body mass index in Korean patients with renal cell carcinoma. J Urol. 2010 Feb;183(2):448-54.

58 Waalkes S, Merseburger AS, Kramer MW, Herrmann TR, Wegener G, Rustemeier J, et al. Obesity is associated with improved survival in patients with organ-confined clearcell kidney cancer. Cancer Causes Control. 2010 Nov;21(11):1905-10.

59 Rogde AJ, Gudbrandsdottir G, Hjelle KM, Sand KE, Bostad L, Beisland C. Obesity is associated with an improved cancer-specific survival, but an increased rate of postoperative complications after surgery for renal cell carcinoma. Scand J Urol Nephrol. 2012 Oct; 46(5):348-57.

60 Zhang J, Chen Q, Li ZM, Xu XD, Song AF, Wang LS. Association of body mass index with mortality and postoperative survival in renal cell cancer patients, a meta-analysis. Oncotarget. 2018 Mar 2;9(17):13959-70.
61 Choueiri TK, Xie W, Kollmannsberger CK, Rini BI, McDermott DF, Knox JJ, et al. The impact of body mass index (BMI) and body surface area (BSA) on treatment outcome to vascular endothelial growth factor (VEGF)targeted therapy in metastatic renal cell carcinoma: results from a large international collaboration. J Clin Oncol. 2010;28(15_Suppl): 4524-4.

62 Steffens S, Grünwald V, Ringe KI, Seidel C, Eggers H, Schrader M, et al. Does obesity influence the prognosis of metastatic renal cell carcinoma in patients treated with vascular endothelial growth factor-targeted therapy? Oncologist. 2011;16(11):1565-71.

63 Ladoire S, Bonnetain F, Gauthier M, Zanetta S, Petit JM, Guiu S, et al. Visceral fat area as a new independent predictive factor of survival in patients with metastatic renal cell carcinoma treated with antiangiogenic agents. Oncologist. 2011;16(1):71-81.

64 Mizuno R, Miyajima A, Hibi T, Masuda A, Shinojima T, Kikuchi E, et al. Impact of baseline visceral fat accumulation on prognosis in patients with metastatic renal cell carcinoma treated with systemic therapy. Med Oncol. 2017 Apr;34(4):47.

65 Martini DJ, Shabto JM, Liu Y, Carthon BC, Speak A, Hitron E, et al. Body mass index (BMI) and toxicities and association with clinical outcomes (CO) in metastatic renal cell carcinoma (mRCC) patients (pts) treated with cabozantinib (cabo). J Clin Oncol. 2019; 37(7_Suppl):613-3.

66 Hakimi AA, Voss MH, Kuo F, Sanchez A, Liu $\mathrm{M}$, Nixon BG, et al. Transcriptomic profiling of the tumor microenvironment reveals distinct subgroups of clear cell renal cell cancer: data from a randomized phase III trial. Cancer Discov. 2019 Apr;9(4):510-25.

67 McDermott DF, Huseni MA, Atkins MB, Motzer RJ, Rini BI, Escudier B, et al. Clinical activity and molecular correlates of response to atezolizumab alone or in combination with bevacizumab versus sunitinib in renal cell carcinoma. Nat Med. 2018 Jun 01;24(6):74957.

68 Cortellini A, Bersanelli M, Buti S, Cannita K, Santini D, Perrone F, et al. A multicenter study of body mass index in cancer patients treated with anti-PD-1/PD-L1 immune checkpoint inhibitors: when overweight becomes favorable. J Immunother Cancer. 2019 Feb 27;7(1):57.

69 Lalani A-KA, Xie W, Flippot R, Steinharter JA, Harshman LC, McGregor BA, et al. Impact of body mass index (BMI) on treatment outcomes to immune checkpoint blockade (ICB) in metastatic renal cell carcinoma (mRCC). J Clin Oncol. 2019;37(7_Suppl): 566-6.

70 De Giorgi U, Procopio G, Giannarelli D, Sabbatini R, Bearz A, Buti S, et al. Association of systemic inflammation index and body mass index with survival in patients with renal cell cancer treated with nivolumab. Clin Cancer Res. 2019 Jul 1;25(13):3839-46. 
71 Bergerot PG, Bergerot CD, Philip EJ, Meza L, Dizman N, Hsu J, et al. Targeted therapy and immunotherapy: effect of body mass index on clinical outcomes in patients diagnosed with metastatic renal cell carcinoma. Kidney Cancer. 2019;3(1):63-70.

72 Labadie BW, Liu P, Bao R, Crist M, Fernandes R, Ferreira L, et al. BMI, irAE, and gene expression signatures associate with resistance to immune-checkpoint inhibition and outcomes in renal cell carcinoma. J Transl Med. 2019 Nov 25;17(1):386

73 Ishihara $\mathrm{H}$, Kondo T, Omae K, Takagi T, Iizuka J, Kobayashi H, et al. Sarcopenia and the modified glasgow prognostic score are signif- icant predictors of survival among patients with metastatic renal cell carcinoma who are receiving first-line sunitinib treatment. Target Oncol. 2016 Oct;11(5):605-17.

74 Yip SM, Heng DY, Tang PA. Review of the interaction between body composition and clinical outcomes in metastatic renal cell cancer treated with targeted therapies. J Kidney Cancer VHL. 2016;3(1):12-22.

75 Cespedes Feliciano EM, Kroenke CH, Caan BJ. The obesity paradox in cancer: how important is muscle? Annu Rev Nutr. 2018 Aug 21;38:357-79.

76 Rajandram R, Perumal K, Yap NY. Prognostic biomarkers in renal cell carcinoma: is there a relationship with obesity? Transl Androl Urol. 2019 May; 8(Suppl 2):S138S46.

77 Hallenborg P, Fiære E, Liaset B, Petersen RK, Murano I, Sonne SB, et al. p53 regulates expression of uncoupling protein 1 through binding and repression of PPAR $\gamma$ coactivator1a. Am J Physiol Endocrinol Metab. 2016 Jan 15;310(2):E116-28.

78 Ginzac A, Barres B, Chanchou M, Gadéa E, Molnar I, Merlin C, et al. A decrease in brown adipose tissue activity is associated with weight gain during chemotherapy in early breast cancer patients. BMC Cancer. $2020 \mathrm{Feb}$ 4;20(1):96 